\title{
The Opacity Project
}

\author{
M.J. Seaton \\ Department of Physics and Astronomy, University College London, \\ Gower St., London WC1E 6BT, UK.
}

\begin{abstract}
The paper gives a brief summary of the work of the Opacity Project.
\end{abstract}

For the purposes of opacity calculations one may divide stellar interiors into two regions: envelopes with mass-densities, $\rho$, less than about $10^{-2} \mathrm{~g} \mathrm{~cm}^{-3}$; and deeper interiors. In stellar envelopes complex atomic systems exist and are not markedly perturbed by the plasma environment. It follows that, for the calculation of envelope opacities, a main need is to have accurate and extensive data for the radiative properties of free atoms and atomic ions. For deeper interiors the nature of the problem changes, one is concerned with simpler radiative processes but plasma perturbations may be of major importance.

The work of the international Opacity Project (to be referred to as OP), has been concerned with the calculation of envelope opacities, which are of particular inportance for studies of stellar pulsations.

The atomic data required are: energy levels; oscillator strengths; photo-ionisation cross-sections; and line-profile parameters. Calculations are made for all cosmicallyabundant elements in all stages of ionisation. The main atomic-physics calculations are made using R-matrix methods and a large team of workers has been involved. Table 1 gives a list of papers on the OP atomic-physics work published in the Journal of Physics B. That work is discussed further by Seaton et al. 1992.

For the calculation of opacities one also requires information concerning the populations of the atomic energy-levels - the problem of the equation of state (EOS). The OP approach is to introduce occupation probabilities $W(i)$ for each level $i$, such that $W(i) \rightarrow 0$ for sufficiently large values of $i$ (highly-excited states), giving convergent partition functions. Table 2 gives a list of papers concerned with the OP EOS work, together with other OP papers published in the Astrophysical Journal.

We find that, to a good approximation for envelopes, the level populations depend only on temperature, $T$, and electron-density, $N_{e}$. The OP opacity calculations are done twice, using codes which are largely independent: firstly in Urbana (Illinois) and Columbus (Ohio) using CRAY-YMP machines at the National Center for Supercomputer Applications of the University of Illinois and at the Supercomputer Center of Ohio State University; and secondly in London using the IBM-3090 at the Rutherford and Appleton Laboratory. Close agreement between the USA and UK results gives us added confidence in their being correct. We compute and archive monochromatic opacities for each chemical element on a grid of $\left(T, N_{e}\right)$-values. These 
monochromatic opacities can then be added for any required chemical mixture and used to calculate Rosseland-mean opacities, $\kappa_{R}$. We obtain tables giving values of $\rho$ and $\kappa_{R}$ as functions of $\left(T, N_{e}\right)$. Interpolation routines give $\kappa_{R}$ for any required values of $\rho$ and $T$. These routines can also be used to produce tables in OPAL format, $\kappa_{R}$ as a function of $T$ for fixed values of $\log (R)$ where $R=\rho / T_{6}^{3}, \rho$ is mass density in $\mathrm{g} \mathrm{cm}^{-2}$ and $T_{6}=10^{-6} \times T$ with $T$ in $\mathrm{K}$.

A full account of the OP opacity work will be given in a paper by M.J. Seaton, Yu Yan, D. Mihalas \& A.K. Pradhan to be submitted to Monthly Notices of the Royal Astronomical Society. The main questions of interest for the present meeting are: how do the OP opacities compare with earlier results from the Los Alamos Opacity Library (LAOL, see Weiss, Keady \& Magee (1990) which gives references to earlier work); and with more recent results from the OPAL project (see Rogers \& Iglesias (1992) and references therein, and the contribution by Rogers in the present volume)? The short answer is that, compared with LAOL, OP gives enhancements at least as large as those postulated by Simon (1982), and that the OP and OPAL results are in good general agreement.

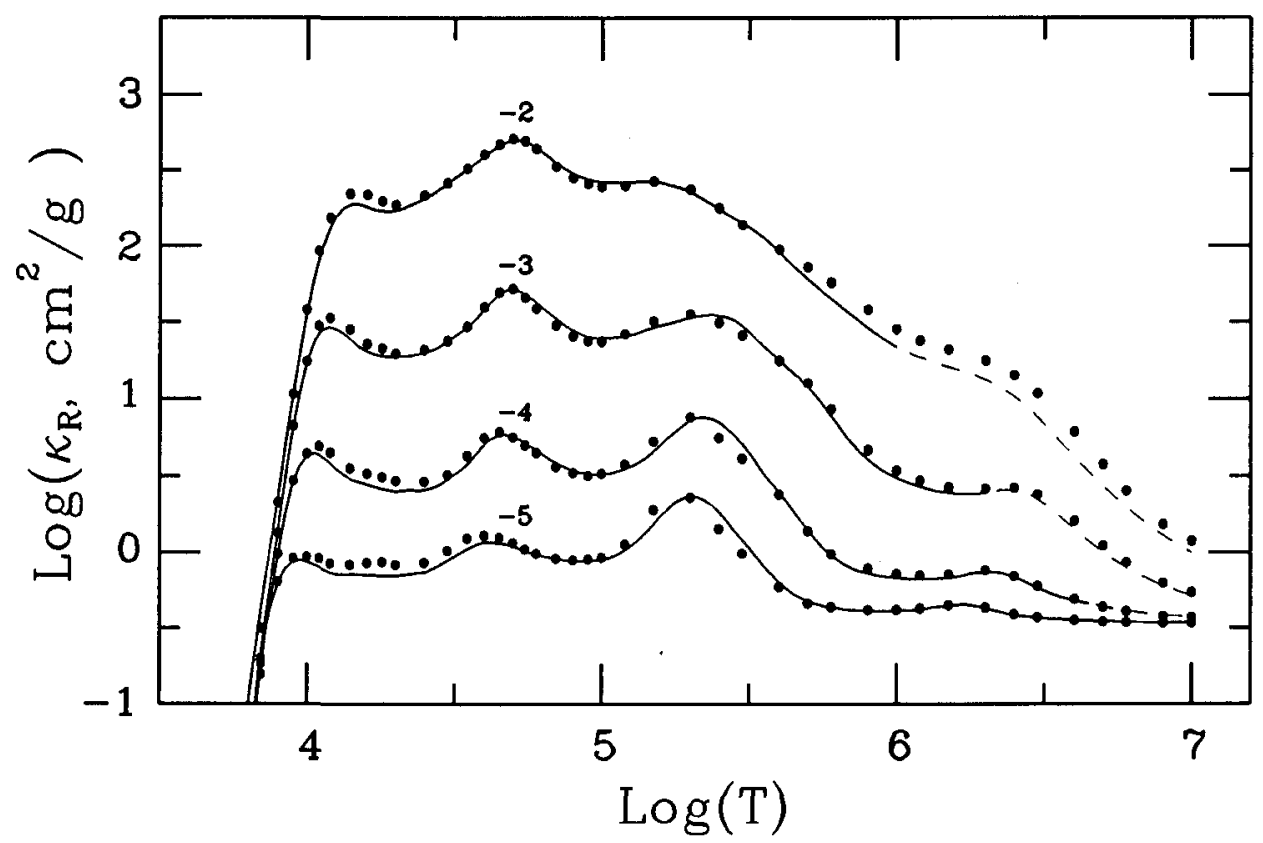

Fig. 1. Rosseland mean opacities for $\mathrm{X}=0.7, \mathrm{Z}=0.02$ and relative metal abundances from Anders \& Grevesse (1989). Full lines from OP, filled circles from OPA. The four curves are for $\log (R)=-1,-2,-3$, and -4 where $R=\rho / T_{6}^{3}$. 
Fig. 1 gives a detailed comparison for just one case, $X=0.7, Z=0.02$ and relative abundances of "metals" from Anders \& Grevesse (1989). For the benefit of users of the opacities I employ the latest results from both opacity projects available at the time of the meeting. Logarithms of Rosseland mean opacities are plotted against $\log (T)$ for four values of $\log (R)$. The following points may be noted.

1. The OP results are plotted with full lines for regions which we define as envelopes, $\log (\rho) \leq-2$, and with dashed lines for higher densities. The OPAL calculations extend to much higher values of $\log (\rho)$.

2. For individual transitions, the atomic data used by OP are more accurate than those used by OPAL. Differences in atomic data may not be so important when one considers the accumulative effects of large numbers of transitions.

3. Both calculations are made with inclusion of fine-structure for transitions in iron. OP uses LSJ coupling while OPAL uses intermediate coupling and hence includes inter-combination lines omitted by OP. At low temperatures $(\log (T)$ of about 4.3), OPAL obtains enhancements, due to inclusion of fine-structure, which are larger than those obtained by OP.

4. The OP calculations have been made with inclusion of the iron-group elements $\mathrm{Cr}, \mathrm{Mn}$ and $\mathrm{Ni}$. These elements, which have abundances much smaller than that of iron, make contributions to opacities which are small but none-the-less significant. OPAL calculations including these elements have been made only for a small number of temperature-density points.

5. Much of the pulsation work is concerned with values of $\log (R)$ in the range -3 to -5 . The main difference between the new opacities, from OPAL and OP, and the old opacities from LAOL is in the magnitude of the maxima which occur at $\log (T) \simeq 5.3$ (the "Z-bump"). The OPAL and OP results are in quite good agreement in that region, although OP gives the maxima shifted to slightly higher temperatures. Some recent calculations show that pulsation results can show surprising sensitivities to the exact values of opacities in the vicinity of the Z-bump.

Other speakers at this meeting discuss the improved agreement between pulsation theory and observations which results from use of the new opacities. A glance at Tables 1 and 2 may convince the reader of the magnitude of the task involved in the opacity work. I think it very fortunate that the new calculations have been made by two entirely independent projects and that the results obtained are in broad agreement. Where there are differences it would be hard to say which results are to be preferred. It may be wise to consider such differences as indicative of the level of uncertainty in both sets of calculations. 
I thank all members of the OP team for their cheerful collaboration. I also thank Carlos Iglesias and Forrest Rogers for many helpful discussions and for providing, in advance of publication, their results used in Figure 1.

\section{References:}

Anders, E. \& Grevesse, N. 1989, Geochim. Cosmochim. Acta, 53, 197.

Rogers, F.J. \& Iglesias, C.A. 1992, Ap. J. Supp., 79, 507.

Seaton, M.J., Zeippen, C.J., Tully, J.A., Pradhan, A.K., Mendoza, C., Hibbert, A. \& Berrington, K.A. 1992, Rev. Mex. Astrn. Astrophys., 23, 19.

Simon, N. R. 1982, Ap. J., 260, L87.

Weiss, A., Keady, J.J. \& Magee, N.H. 1990, Atomic Data Nucl. Data Tables, 45, 209.

\section{DISCUSSION}

C.A. IGLESIAS: The uncertainties in the abundances are likely to overwhelm the $20 \%$ discrepancies between the OP and OPAL results.

M.J. SEATON: True. But we can always hope that the accuracy of abundances will improve.

N.R. SIMON: How much more are the opacities likely to increase?

M.J. SEATON. Our sins may be of omission rather than of commission. One can go on thinking of processes which should be included and which may lead to further opacity increases. But I do not think that we can give you any further factors of 3 or more.

D. WELCH: Are monochromatic opacity tables available on request?

M.J. SEATON: In principle, yes. But beware, the amounts of data may be very large, despite our "packing" of the archived files (we use the minimum number of frequency points which allow linear interpolations to some prescribed accuracy, usually taken to be 1 or $2 \%$ ).

R. STELLINGWERF: Do you expect the Rosseland mean opacities to be "smooth" in temperature and density? This is a basic assumption when interpolating in tables.

M.J. SEATON: So far as the tables themselves are concerned, the question of "smoothness" does not arise. It all depends on the interpolation procedure used. Cubic splines will give smoothness in the functions and first derivatives. Something better may be needed. I intend to give some thought to that. 
Table 1. OP papers published in the Journal of Physics B.

Papers in the series "Atomic data for Opacity Calculations":-

I. Seaton, General formulation. 20, 6363, 1987.

II. K.A.Berrington, P.G.Burke, K.Butler, M.J.Seaton, P.J.Storey, K.T.Taylor \& Yu Yan, Computational Methods. 20, 6379, 1987.

III. Yu Yan, K.T.Taylor \& M.J.Seaton. Oscillator strengths for C II. 20, 6399, 1987.

IV. Yu Yan \& M.J.Seaton. Photoionisation cross sections for C II. 20, 6409, 1987.

V. M.J.Seaton. Electron impact broadening of some C III lines. 20, 6431, 1987.

VI. J.F.Thornbury \& A.H.Hibbert. Static dipole polarisabilities of the ground states of the helium sequence. 20,6447, 1987.

VII. J.A.Fernley, K.T.Taylor \& M.J.Seaton. Energy-levels, f-values and photo-ionisation cross sections for He-like ions. 20, 6457, 1987.

VIII. M.J.Seaton. Line-profile parameters for 42 transitions in Li-like and Be-like ions. 21, 3033, 1988.

IX. G.Peach, H.E.Saraph \& M.J.Seaton. The lithium iso-electronic sequence. 21, $3669,1988$.

X. D.Luo, A.K.Pradhan, H.E.Saraph, P.J.Storey \& Yu Yan. Oscillator strengths and photoionisation cross sections for O III. 22, 389, 1989.

XI. D.Luo \& A.K.Pradhan. The carbon iso-electronic sequence. 22, 3377, 1989.

XII. M.J. Seaton, Line-profile parameters for neutral atoms of He, C, N and O. 22, $3603,1989$.

XIII. M.J. Seaton. Line profiles for transitions in hydrogenic ions. 23, 3255, 1990.

XIV. J.A.Tully, M.J.Seaton \& K.A.Berrington. The berrylium sequence. 23, 3811, 1990.

XV. P.M.J. Sawey \& K.A. Berrington, Fe I to IV. 25, 1451, 1992.

XVI. H.E. Saraph, P.J. Storey \& K.T. Taylor, $A b$ initio calculations for Fe VIII and Fe VII. In press.

Other papers:-

- M.J. Seaton, On the R-matrix method for bound-state calculations: I. General theory. 18, 2111, 1985.

- K.A. Berrington \& M.J. Seaton. On the R-matrix method for bound-state calculations. II Results for energy levels of $\mathrm{C} ;+.18,2587,1985$.

- M.J. Seaton, Outer-region contributions to radiative transition probabilities. 19, 2601, 1986. 
Table 2. OP papers published in the Astrophysical Journal

Papers in the series "Equations of State for Stellar Envelopes":-

I. D.G. Hummer \& D. Mihalas. An occupation probability formalism for the truncation of the internal partition function. 331, 794, 1988.

II. D. Mihalas, W. Däppen \& D.G. Hummer. Algorithms and selected results. 331, 815, 1988.

III. W. Däppen, D. Mihalas, D.G. Hummer \& B.W. Mihalas. Thermodynamic quantities. 332, 261, 1988.

IV. D. Mihalas, D.G. Hummer, B.W. Mihalas \& W. Däppen. Thermodynamic quantities and selected ionization fractions for six elemental mixes. 350, 300, 1990.

Other papers:-

- Däppen, W., Anderson, L.S. \& Mihalas, D. "Statistical mechanics of partially ionised stellar plasmas", 319, 195, 1987.

- D.G. Hummer, "A fast and accurate method for evaluating the nonrelativistic free-free Gaunt factor for hydrogenic ions", 327, 477, 1988. 